\title{
Anterior cervical spine surgery for the treatment of subaxial cervical spondylodiscitis: a report of $\mathbf{3 0}$ consecutive patients
}

\author{
Benedikt W. Burkhardt, MD, Simon J. Müller, MD, Anne-Catherine Wagner, MD, and \\ Joachim M. Oertel, MD
}

Department of Neurosurgery, Saarland University Medical Center and Saarland University Faculty of Medicine, Homburg-Saar, Germany

OBJECTIVE Infection of the cervical spine is a rare disease but is associated with significant risk of neurological deterioration, morbidity, and a poor response to nonsurgical management. The ideal treatment for cervical spondylodiscitis (CSD) remains unclear.

METHODS Hospital records of patients who underwent acute surgical management for CSD were reviewed. Information about preoperative neurological status, surgical treatment, peri- and postoperative processes, antibiotic treatment, repeated procedure, and neurological status at follow-up examination were analyzed.

RESULTS A total of 30 consecutive patients (17 male and 13 female) were included in this retrospective study. The mean age at procedures was 68.1 years (range 50-82 years), with mean of 6 coexisting comorbidities. Preoperatively neck pain was noted in 21 patients (70.0\%), arm pain in $12(40.0 \%)$, a paresis in $12(40.0 \%)$, sensory deficit in $8(26.7 \%)$, tetraparesis in $6(20 \%)$, a septicemia in 4 (13.3\%). Preoperative MRI scan revealed a CSD in one-level fusion in 21 patients $(70.0 \%)$, in two-level fusions in 7 patients $(23.3 \%)$, and in three-level fusions in 2 patients $(6.7 \%)$. In 16 patients an antibiotic treatment was initiated prior to surgical treatment. Anterior cervical discectomy and fusion with cervical plating (ACDF+CP) was performed in 17 patients and anterior cervical corpectomy and fusion (ACCF) in 12 patients. Additional posterior decompression was performed in one case of ACDF+CP and additional posterior fixation in ten cases of ACCF procedures. Three patients died due to multiple organ failure (10\%). Revision surgery was performed in 6 patients (20.7\%) within the first 2 weeks postoperatively. All patients received antibiotic treatment for 6 weeks. At the first follow-up (mean 3 month) no recurrent infection was detected on blood workup and MRI scans. At final follow-up (mean 18 month), all patients reported improvement of neck pain, all but one patients were free of radicular pain and had no sensory deficits, and all patients showed improvement of motor strength. One patient with preoperative tetraparesis was able to ambulate.

CONCLUSIONS CSD is a disease that is associated with severe neurological deterioration. Anterior cervical surgery with radical debridement and appropriate antibiotic treatment achieves complete healing. Anterior cervical plating with the use of polyetheretherketone cages has no negative effect of the healing process. Posterior fixation is recommended following ACCF procedures.

https://thejns.org/doi/abs/10.3171/2018.10.FOCUS18464

KEYWORDS anterior cervical procedures; cervical spondylodiscitis; cervical spine; clinical outcome; epidural empyema; reoperation

$\mathrm{P}$ YOGENIC spinal infection has affected human beings since ancient times. The reported incidence varies from 1:250,000-1:400,000 and the most commonly affected region is the lumbar and thoracic spine..$^{5,10,18}$ Infection of the cervical spine is rare and treatment strategies for infections of the cervical spine and cervical spondylodiscitis (CSD) may vary. ${ }^{15,30}$

Before the advent of antibiotics, the mortality rate of spinal infections was almost $70 \%$ and is still up to $16 \%$ within the first year in spite of surgical and antimicrobial

ABBREVIATIONS ACCF = anterior cervical corpectomy and fusion; $A C D F=$ anterior cervical discectomy and fusion; $A C D F+C P=A C D F$ with cervical plating; $B M I=$ body mass index; CRP = C-reactive protein; CSD = cervical spondylodiscitis; ICU = intensive care unit; PEEK = polyetheretherketone.

SUBMITTED August 31, 2018. ACCEPTED October 22, 2018.

INCLUDE WHEN CITING DOI: 10.3171/2018.10.FOCUS18464. 
treatment. . $8,9,15,26$ Aging of the Western civilization and drug abuse are causes for an increasing incidence of spinal infections attributed to rising rates of comorbidities. ${ }^{2,22,31}$ Most recently an increasing incidence of CSD and an increased mean age at surgery have been reported. ${ }^{31}$

The surgical treatment of CSD remains challenging since the integrity of ligamentous and osseous structures of the cervical spine might be affected intraoperatively. Adequate treatment is mandatory because CSD manifests rapidly, and progression is often followed by neurological decline that results in tetraparesis, sepsis, and death. Mortality rates of up to $21 \%$ have been reported in the literature. ${ }^{28,35}$ Furthermore, there is no consensus about the ideal timing or optimal surgical technique for the treatment of CSD. Despite the reports of several authors who demonstrated the successful insertion of titanium mesh cages with and without posterior instrumentation, ${ }^{17,27}$ and other authors who reported the successful insertion of a stand-alone polyetheretherketone (PEEK) cage,$^{20}$ not all spine surgeons are likely of the opinion that implantation of nonautogenic material is still adequate.

Some authors have reported bacterial colonization of the implant material and therefore do not recommend the use of cervical plates. ${ }^{29,30}$ Additionally there is no consensus or guideline regarding the duration of antibiotic treatment. Due to the rare nature of this disease, there are a limited number of studies that focus on CSD with more than 30 patients.

The present article describes the clinical course of 30 consecutive patients who underwent anterior cervical spine procedures for CSD. Special focus was given to the surgical strategy, postoperative treatments, and clinical outcome of this cohort.

\section{Methods}

Patient Population, Clinical, and Radiographic Parameters

All patients who underwent surgical treatment for subaxial (C3-T1) CSD from January 2011 to June 2017 at a neurosurgical department were retrospectively identified using an electronic file database. Patients' files and outpatient visit reports were reviewed with focus on the following data: age, body mass index (BMI), medical history and comorbidity, American Society of Anesthesiologists, or ASA, score at the time of surgical treatment for CSD, duration of symptomatology, the presence of pain and a neurological deficit, duration of antibiotic treatment prior to the procedure, number of operated levels, duration between MRI- or CT-based diagnosis and surgical treatment, duration of stay at the intensive care unit (ICU) postoperatively, peri- and postoperative presence of inflammation parameters (C-reactive protein [CRP]), intraoperative microbiological findings, and organism cultured.

Additionally, intra- and perioperative data, such as specific surgical techniques and surgical complications, including incidental durotomy, damage of nerve structures, cerebrospinal fluid leakage, wound infection, and implantrelated complications, were assessed.

Furthermore, the preoperative, postoperative and follow-up imaging studies of the cervical spine (i.e., MR images, CT scans, and radiographs) was reviewed. The pres- ence of an epidural empyema and involvement of the spinal cord were noted.

The preoperative diagnosis of CSD was based on the clinical symptoms, hematological findings, and radiological changes in the disc space and vertebral body.

The diagnosis was confirmed by intraoperative findings such as the presence of purulent disc material, destroyed osseous structures, and soft-tissue inflammation.

Indications for surgery were intractable neck pain, septicemia, epidural empyema, neurological deficits and deterioration of neurological deficits, and persistent infection that did not respond to antibiotic treatment.

\section{Surgical Strategy and Initial Surgical Treatment}

The surgical strategy for the treatment of CSD consisted of an anterior approach for adequate surgical debridement, reconstruction of the disc space and osseous defect, and restoration of adequate stability and cervical alignment.

Once the disc space was exposed, a total discectomy was performed for removal of the purulent material. The specimen that was extracted during surgery was sent for microbiological examination. Next, the endplates of the two adjacent vertebrae were inspected. An empty PEEK cage was used for ACDF procedures. In general an $\mathrm{ACDF}+\mathrm{CP}$ was performed once there was no sign of lost osseous integrity and no inflammatory infestation of the vertebral body. In case of osseous destruction of the vertebral body or poor bone quality, the decompression was extended, and a full corpectomy was performed to achieve ACCF using a cylindrical expandable cage with additional plating. Monocortical screw placement of the cervical plating was performed in all cases. In general ACCF procedure was followed by posterior instrumentation.

Thirteen single-level ACDF procedures were performed, and in 10 patients additional cervical plating was placed. In one case, $\mathrm{ACDF}+\mathrm{CP}$ was followed by posterior laminectomy for evacuation of epidural empyema at two different levels. In two patients additional lumbar spine surgery was performed for lumbar spondylodiscitis. Four two-level $\mathrm{ACDF}+\mathrm{CP}$ procedures were performed.

A two-level ACCF was performed in 10 patients and combined with posterior fixation in 7 of the 10 . Threelevel ACCF procedures combined with posterior fixation were performed in two patients. One patient died during the preoperative course.

\section{Statistical Analysis}

The SPSS statistical software package (IBM Corp.) was used for statistical analysis of the data. We used the Fisher exact test to compare relative frequencies of a binary outcome and an outcome with more than 2 categories between 2 independent groups, respectively.

The Wilcoxon test was used to compare nonparametric paired-sample tests, and the Mann-Whitney U-test was applied for comparison of a quantitative non-normal outcome between 2 groups. Any p values given were 2 -sided. $\mathrm{p}<0.05$ was assumed to be sufficient to indicate statistical significance. 


\section{Results}

\section{Patient Characteristics}

Thirty consecutive patients (17 male and 13 female) were identified from the surgical database. The mean age at surgery was 68.1 years (range 50-82 years). All patients were referred to our hospital from another institution. The mean BMI was 27.9; 16 patients $(53.3 \%$ ) had a BMI ranging from 25.0 to 29.9 , and 7 patients (23.3\%) had a BMI of over 30.0. The mean number of coexisting comorbidities was 5 . A compilation of patient demographics, coexisting medical conditions, diseased segments, surgical technique, antibiotic treatment, and infection parameters is shown in Table 1.

In 16 cases an antibiotic treatment was initiated for treatment of fever and infection of unknown focus prior to diagnostic MRI and surgical treatment.

The mean duration of symptoms prior to surgical treatment was 30 days (range 1-180 days). The mean time from initial MRI scan with the suspected diagnosis of a CSD to transferal in our neurosurgical department was 2 days (range less than 1-18 days). In 19 patients the surgical procedure was indicated on the day of admission due to a debilitating general condition and progressive neurological deterioration. The mean time from admission to the neurosurgical department to surgical treatment was 36 hours (range less than 1-6 days).

\section{Preoperative Clinical Findings and Preoperative MRI and CT Presentation}

A compilation of clinical presentations prior to surgery and at final follow-up is shown in Table 2. Two patients were ventilator dependent: in one case due to myelitis (Fig. 1), whereas in the other case it was due to coexisting medical disease and poor general condition.

According to the ASA classification system, 9 patients had grade 2 status, 18 patients had grade 3 status, and 2 patients had grade 4 status. One patient who was given grade of 5 died during the preoperative preparations. A compilation of preoperative imaging findings in each case is shown in Table 1.

In 1 of the patients with no destruction on the vertebral endplate, subsidence of an expandable cylindrical cage occurred and a revision procedure was performed (reoperation 4).

In 2 of the patients with loss of vertebral endplate integrity and signs of erosion, a repeated procedure was performed for implant dislocation with consecutive destruction of the vertebral body (reoperations 2 and 3 ).

In 3 of 5 of the patients with a complete or a partial destruction of the vertebral body, a repeated procedure was performed: in 1 patient for subsidence of the expandable cylindrical cage, in 1 patient for mass lateral screw malpositioning, and in 1 patient for wound healing problems (reoperations 1,2, and 6).

\section{Complications and Reoperations}

Six patients underwent a revision procedure. The indications were as follows.

\section{Subsidence of the Expandable Cylindrical Cage}

Two weeks after a 2-level ACCF without posterior in- strumentation, the patient complained about acute dysphagia. A CT-scan revealed subsidence of the expandable cylindrical cage into the cranial adjacent vertebral body, pullout of the cranial screw, and dislocation of the cervical plate. The patient underwent a revision procedure. Corpectomy of the cranial vertebral body and a consecutive 3-level ACCF with additional posterior lateral mass stabilization were performed.

\section{Implant Loosening}

Pullout of the caudal and cranial screws with cage dislocation and destruction of the caudal vertebral body was observed following a single-level $\mathrm{ACDF}+\mathrm{CP}$. The patient underwent 2-level ACCF with lateral mass stabilization 6 days after the initial procedure.

\section{Cage Dislocation}

Postoperative dysphagia due to cage dislocation was observed following a single-level ACDF. The patient required revision surgery, and a single-level $\mathrm{ACDF}+\mathrm{CP}$ was performed 6 days after the initial procedure.

\section{Subsidence of the Expandable Cylindrical Cage}

Destruction of the caudal vertebral body due to subsidence of the expandable cylindrical cage following a 2-level ACCF was observed. The patient underwent a 3-level ACCF with lateral mass stabilization on the 4th postoperative day (see Fig. 2).

\section{Screw Malpositioning}

One patient underwent revision procedure following 2-level ACCF with lateral mass stabilization due to radicular pain caused by lateral mass screw misplacement.

\section{Wound Healing}

In this case, a posterior wound-healing problem occurred following a 2-level ACCF with lateral mass stabilization, and a revision procedure was performed during the postoperative period.

\section{Antibiotic Treatment and Postoperative Course}

In 19 patients a stay of more than 2 days in the ICU was required. Nine patients had to stay between 3-5 days, 6 patients had to stay between 6-14 days, and 4 patients had to stay more than 14 days. In none of the surgically treated patients was external immobilization via collar, orthosis, or halo performed.

The concurrent existence of an epidural empyema had no statistical difference in terms of duration in the ICU (Mann-Whitney U-test, $\mathrm{p}=0.556$ ), but all patients who initially presented with a tetraparesis and who died in this series had a coexisting epidural empyema.

Two multimorbid patients died due to multiple organ failure in the early postoperative period (6.9\%). A doubleantibiotic therapy was initiated in all patients with unknown microbial organism. One or more bacteriological specimens were identified in the preoperatively acquired microbiological cultures and the intraoperatively obtained smears in 20 patients $(69.0 \%)$.

Once a bacteriological specimen was identified and an 


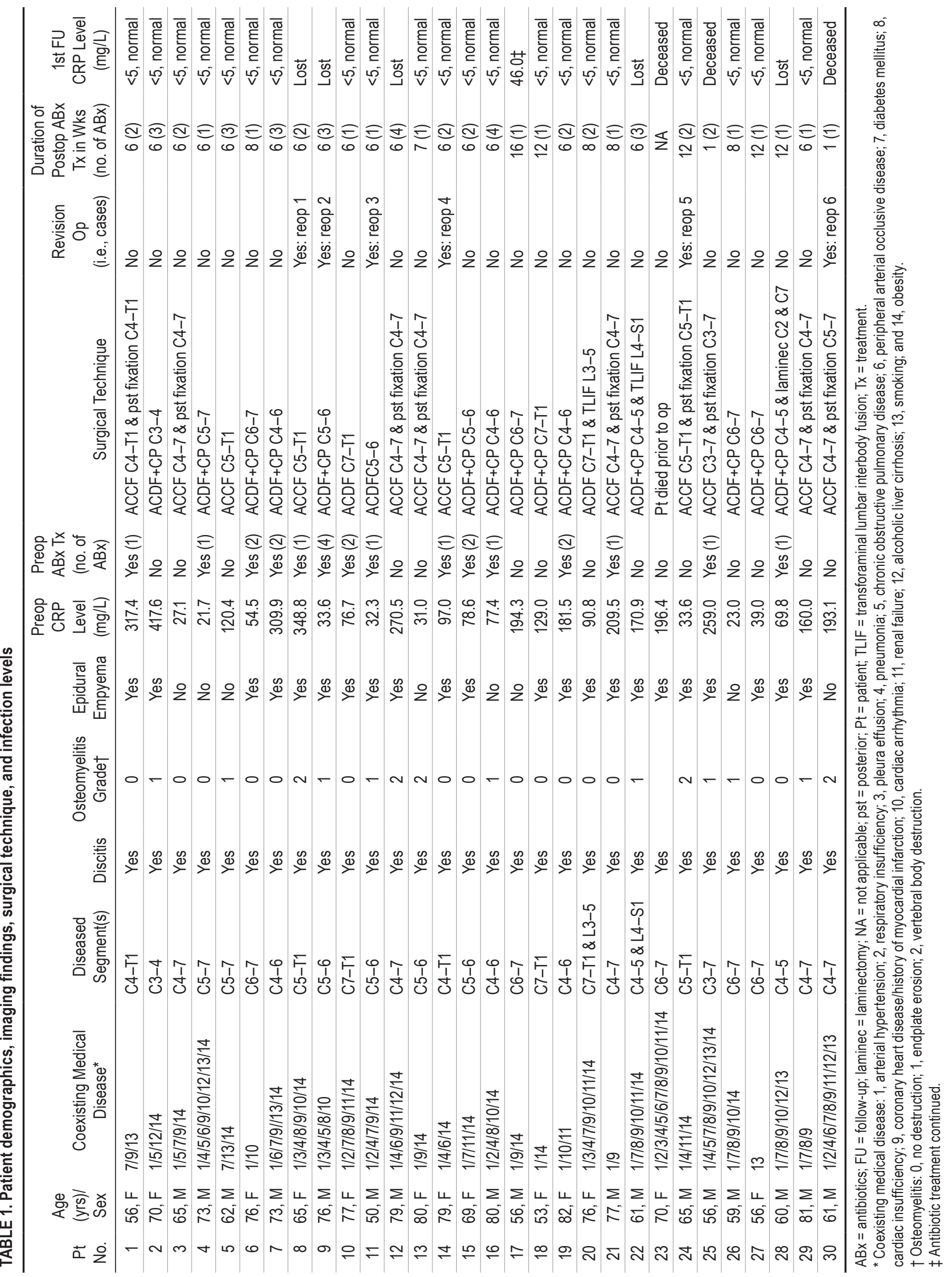




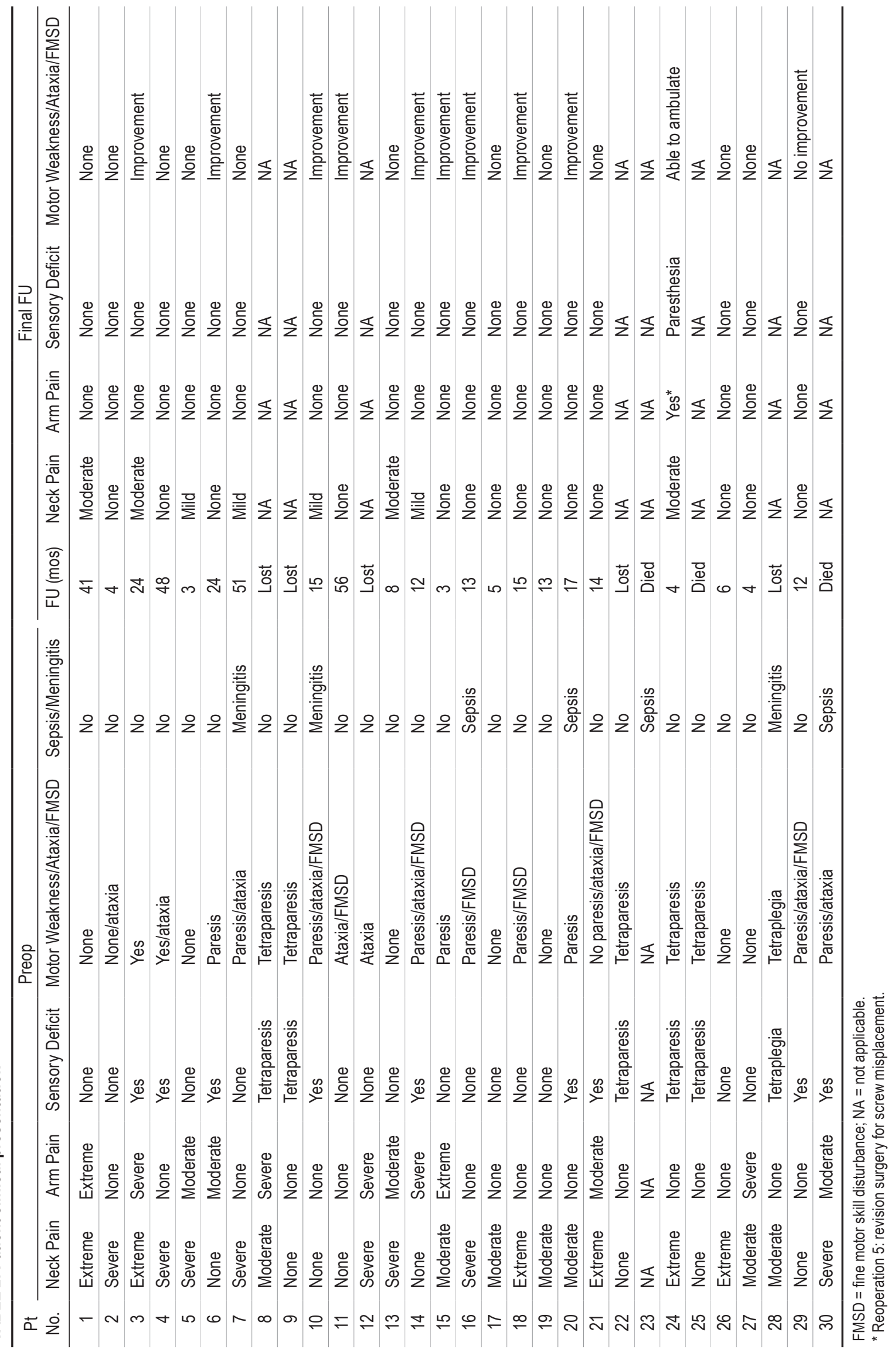



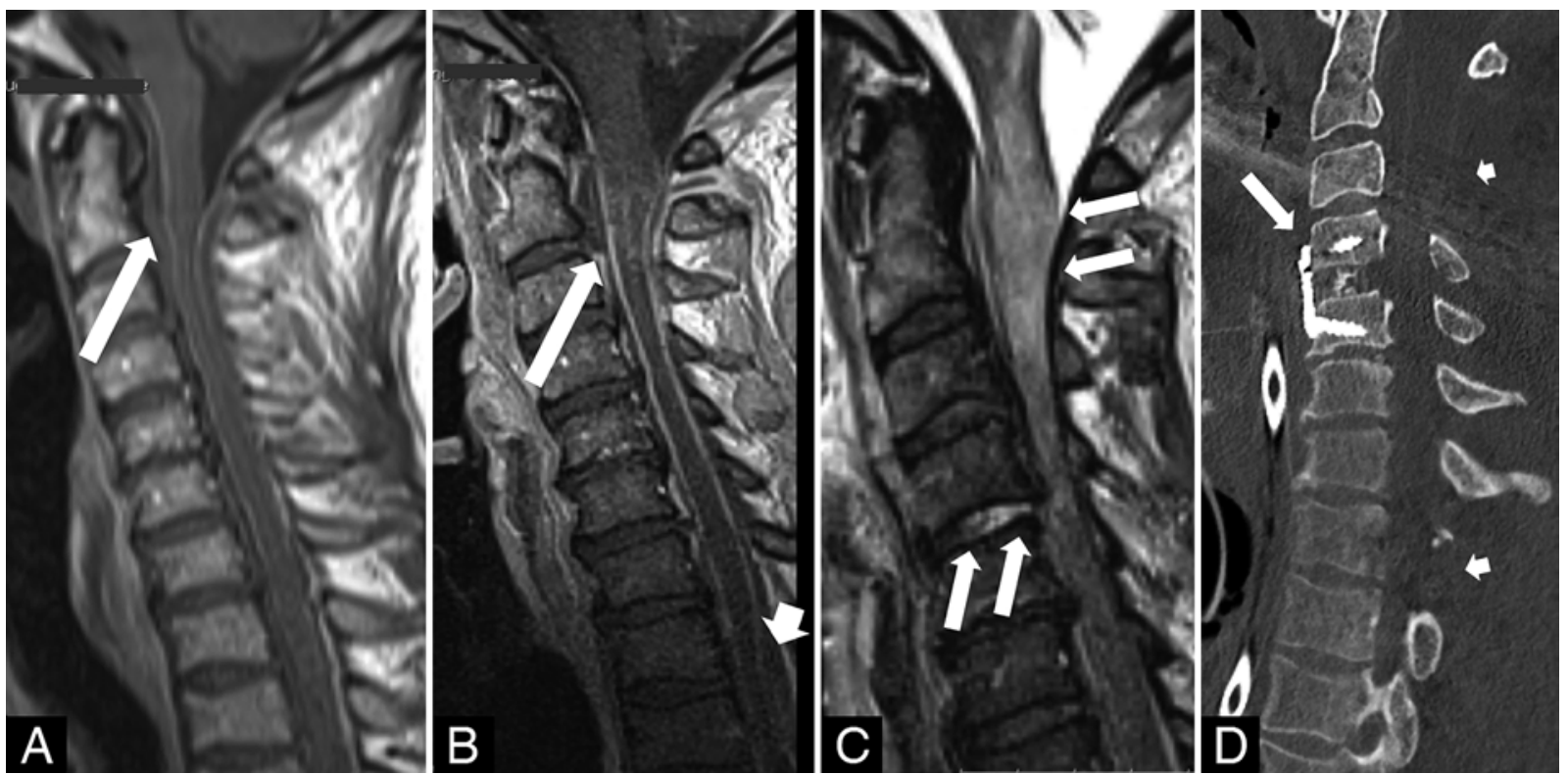

FIG. 1. Case 1. Preoperative MRI and postoperative CT images obtained in a 60-year-old man with neck pain and fever who was admitted to an outside hospital. A: MR image with contrast enhancement at admission showing an epidural empyema at C2-3 (arrow). An antibiotic treatment was initiated at this time. B: MR image (prior to transferal to our department), obtained after the patient became septic and ventilator dependent, showing contrast enhancement along the spinal canal (arrow) and empyema at C7-T1 (arrowhead). C: MR image showing an edema of the medulla oblongata and spinal cord due to myelitis and a CSD at C4-5 (small arrows). D: Postoperative CT scan showing the result of ACDF+CP (arrow) and posterior decompression at C2 and T1 (arrowheads).

antibiogram was carried out, the antibiotic treatment was adapted accordingly. A summary of the bacteriological specimens is shown in Table 3.

In all patients an antibiotic treatment was carried out for at least 6 weeks. The antibiotic treatment was extended up to 3 month in case of a multidrug antimicrobial-re- sistant specimen such as methicillin-resistant Staphylococcus aureus. The intravenous antibiotic treatment was performed till CRP levels were normal $(<5 \mathrm{mg} / \mathrm{L})$ before antibiotic treatment was continued orally for the required period.

The average hospitalization duration was 19 days (range
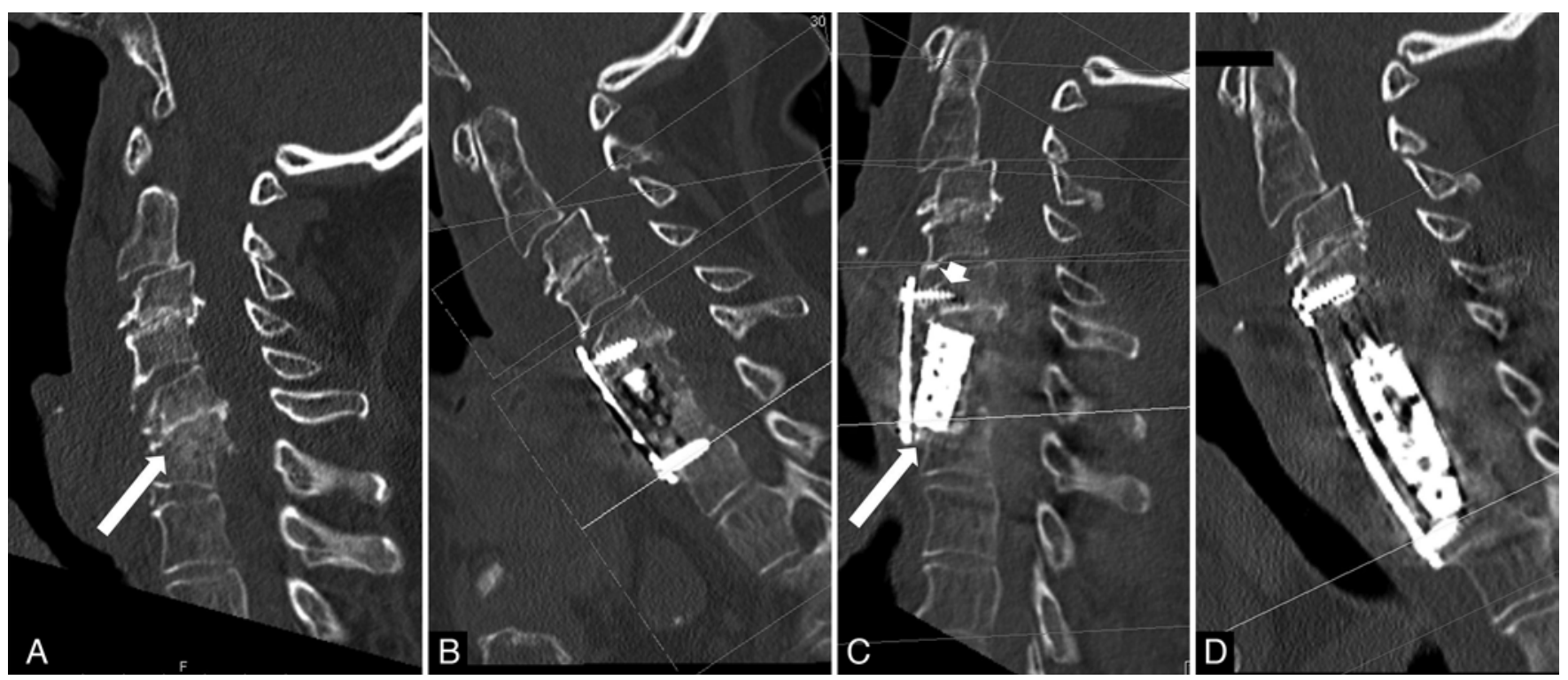

FIG. 2. Reoperation no. 4. A: Preoperative CT scan showing vertebral body destruction (arrow). B: Postoperative CT scan after 2-level ACCF. C: Additional CT scan after sudden onset of dysphagia with subsidence of the expandable cylindrical cage (arrow), the beginning of screw loosening, and dislocation of the cervical plate (arrow tip). D: CT scan after reoperation with additional posterior fixation. 
TABLE 3. Bacteriological findings

\begin{tabular}{lc}
\hline Bacteriological Specimen & No. of Cases \\
\hline Staphylococcus aureus & 10 \\
\hline Staphylococcus epidermidis & 4 \\
\hline Methicillin-resistant S. aureus & 4 \\
\hline Streptococcus agalactiae & 1 \\
\hline Enterococcus faecalis & 1 \\
\hline Escherichia coli & 1 \\
\hline Klebsiella oxytoca & 1 \\
\hline Candida albicans & 1 \\
\hline
\end{tabular}

4-37 days), and the CRP level decreased on average by $69.8 \%$ during the time of hospitalization. Sixteen patients were referred to a rehabilitation center and 5 patients to a paraplegia center.

\section{Follow-Up Visits}

The first follow-up included a control of CRP value and MRI scan of the cervical spine. Generally it was about 1 to 2 weeks after ending of the antibiotic therapy. The average time from surgical treatment to first follow-up was 3 month; 5 patients did not present for follow-up visits. Healing was defined once there was no elevated CRP level and no signs of infection on the MRI scan.

At first follow-up, the CRP value was in the normal range in 21 of 22 patients. In 1 patient because the CRP value was slightly elevated, antibiotic therapy was continued. There was no evidence of latent or progressive infection on the MRI scan in all cases.

Of 8 patients who complained of neck pain, 4 reported moderate neck pain and 4 patients reported mild neck pain. One patient who underwent revision surgery for lat- eral mass screw misplacement reported radicular pain as unchanged. One of the patients with preoperative tetraparesis was able to ambulate. All patients with preoperative paresis had a complete recovery or improvement of muscle strength. In all except 1 patient, a complete recovery or improvement of sensory deficit was noted. These results remained stable in all patients until the last follow-up (average follow-up 18 months). No recurrent infection of the spinal column occurred. A compilation of preoperative and final follow-up clinical findings for each patient is shown in Table 2. For demonstration purposes 2 cases (case 2 and case 3 ) with preoperative and follow-up imaging are shown in Figs. 3-6.

\section{Discussion}

In the Western civilization, nonspecific infections of the cervical spine are more frequent than specific infections. In general, nonspecific spondylodiscitis is caused by bacteria and affects elderly people or people with a weakened immune system. There are various risk factors for a weakened immune system, such as diabetes mellitus, pulmonary and urogenital infections, rheumatic disease, immunosuppressive diseases, immunosuppressive therapies such as steroid application, and intravenous drug abuse. $1,33,37$ Since elderly patients often have multiple risk factors, they are more likely to suffer from an infection of the cervical spine. The mean age of patients in the present study was 68.1 years and is slightly older than in other reports. ${ }^{6,11,13}$ This might explain why the mean number of coexisting morbidities in the present cohort was considerably higher than in other cohorts..$^{6,11,13}$

The incidence of epidural empyemas in the present series (i.e., $73.3 \%$ ) was in accordance with the rate reported in the literature (i.e., 60\%-100\%). ${ }^{11,13,31}$ The compression of the nerve structures by the epidural empyema was the
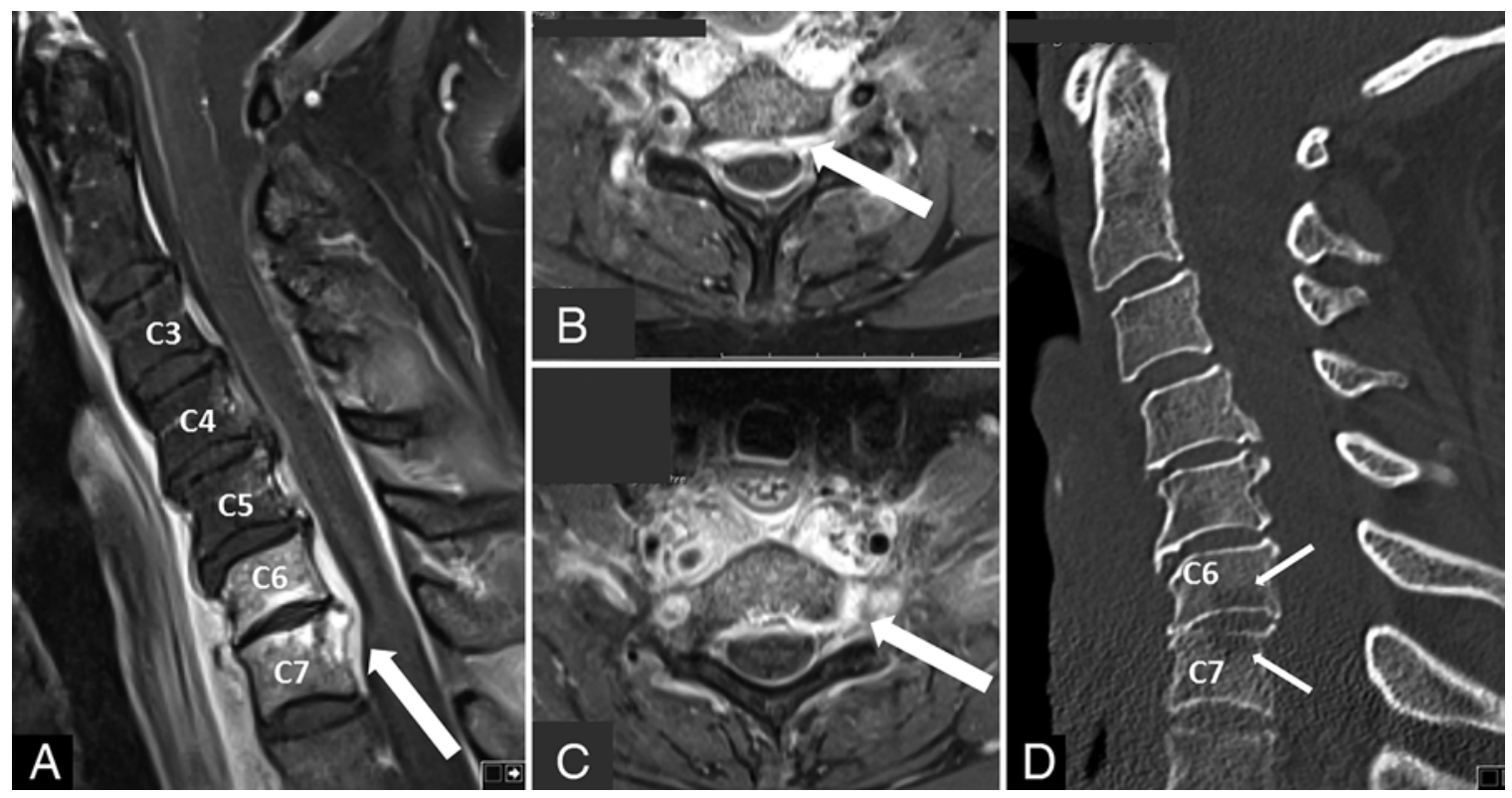

FIG. 3. Case 2. Preoperative MRI and CT images. A: Cervical spondylodiscitis at C6-7 with an epidural empyema (arrow). B: Intraspinal epidural empyema (arrow). C: Compression of a left-sided C7 nerve root (arrow). D: Loss of bone density of C6 and C7 vertebral bodies (arrows). 

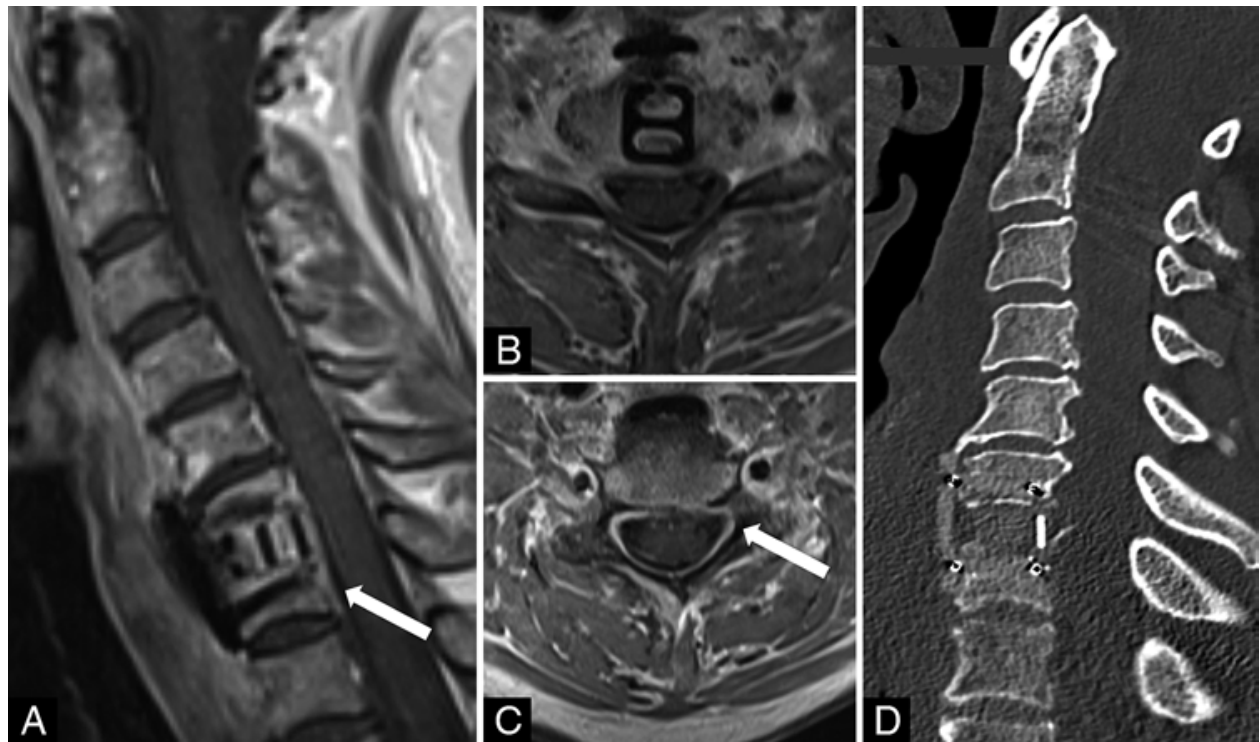

FIG. 4. Case 2. Postoperative 3-month follow-up MR images. A: No contrast enhancement and no sign of epidural empyema. B: No sign of ongoing infection. C: Nerve root and neural foramen free of compression. Note the neural foramen of the C7 nerve root (arrow). D: No implant subsidence, and the vertebral body endplates are intact.

cause of paresis of the upper extremity in 8 patients, for tetraparesis in 6 , and was present in 3 of those who passed away, as described above.

The mean duration of symptoms prior to establishing the diagnosis was 21 days in patients with a CSD and epidural empyema and therefore was shorter than that of patients without coexisting epidural empyema (mean 54 days). The ideal treatment strategy for spinal infections remains unclear. Some authors believe that a diagnosis can be established faster due to improved imaging modalities and recommend conservative treatment for spinal infections of up to 6 weeks. ${ }^{4,9,16,32}$
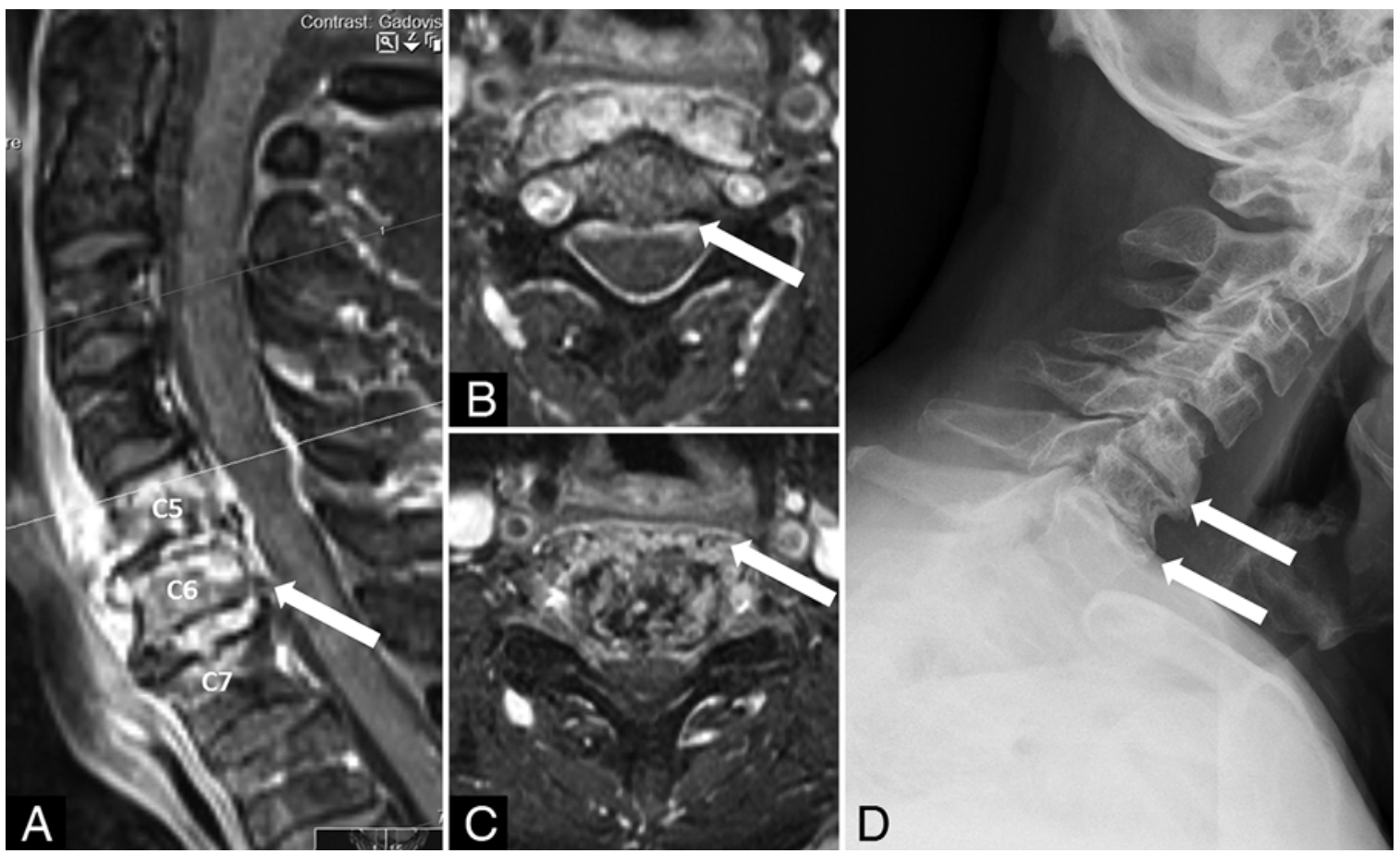

FIG. 5. Case 3. Preoperative MR images and a radiograph of the cervical spine. A: Two-level C5-6 and C6-7 CSD with collapse of the disc space and epidural empyema (arrow). B: Intraspinal empyema (arrow). C: Prevertebral signs of infection (arrow). D: Ventral spondylophyte formation and collapse of the disc spaces at C5-6 and C6-7 (arrows). 

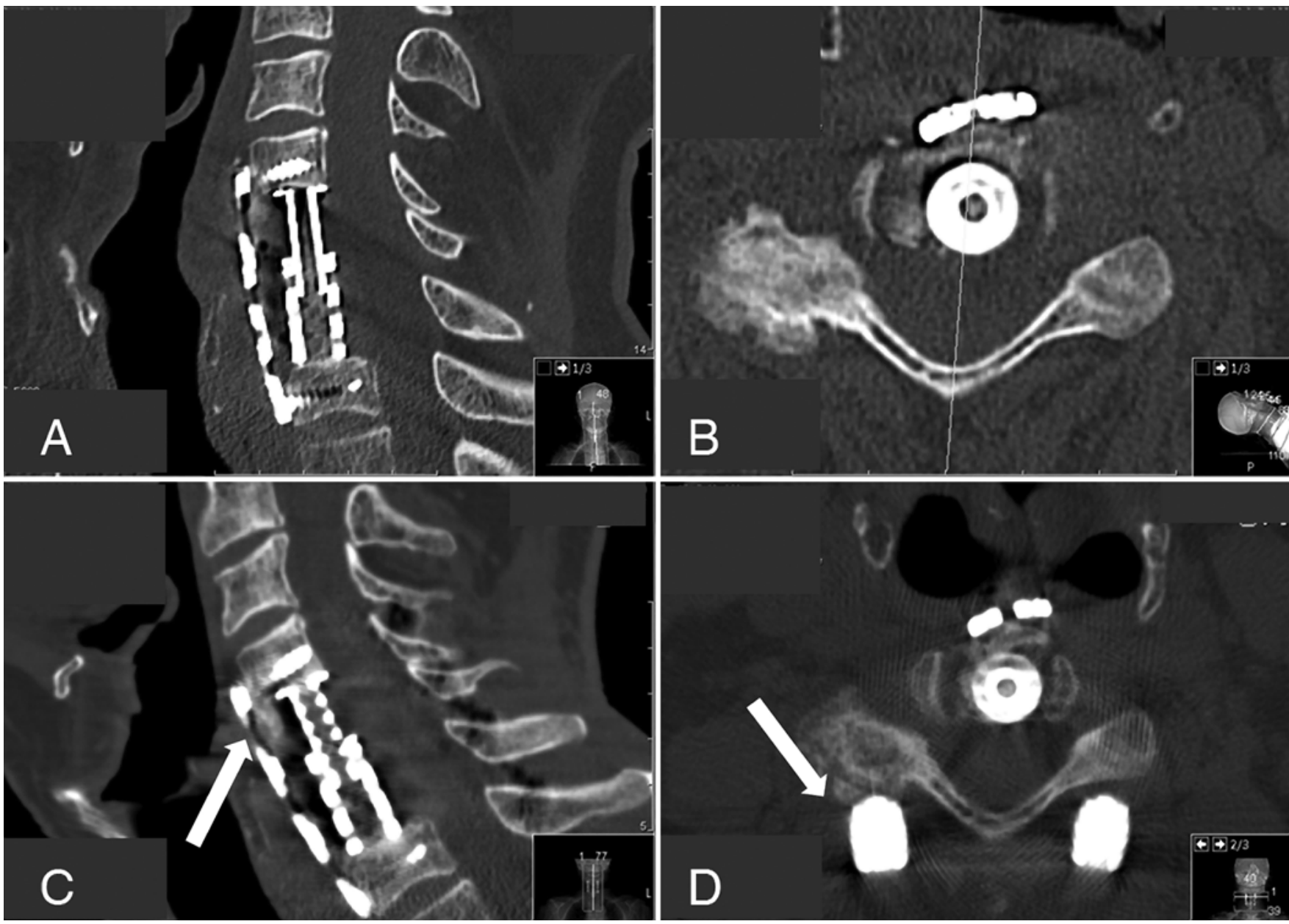

FIG. 6. Case 3. Postoperative and 1-year follow-up CT scans. A and B: Two-level ACCF using an expandable cage and additional cervical plating. C: Unchanged implant positioning with signs of osseous formation (arrow) and integration of the expandable cylindrical cage in the endplate of the cranial vertebral body. D: Unchanged positioning of the expandable cylindrical cage, head of a lateral mass screw (white arrow). Each inset is an expandable cylindrical cage placed after the corpectomy was performed. The official name of the product is ADD (Ulrich Medical).

Other authors consider surgical treatment followed by systemic antibiotic treatment as the gold standard since the neurological status of patients might deteriorate considerably while receiving antibiotic treatment. ${ }^{7,8,14,25}$

In the present study the onset of unspecific clinical symptoms varied from 1 day to 6 months prior to transferal to our department, and in 16 patients (53.3\%) an antibiotic therapy regimen had already been initiated. However, 10 of those 16 patients who had already been receiving an antibiotic treatment deteriorated and developed paresis and tetraparesis.

It has been reported that infections of the cervical spine are associated with a more rapid neurological deterioration than infections of the lumbar and thoracic spine..$^{28,34,35}$ The findings of our study are consistent with these reports since the mean time between initial unspecific clinical symptoms and the occurrence of a motor deficit was 22 days. Therefore, we are of the opinion that particularly patients with infections of the cervical spine who receive antibiotic treatment should be evaluated closely with respect to their neurological status.
It remains unclear if early surgery is superior to delayed surgery in cases of infections of the cervical spine. The retrospective design of our study prohibits any profound conclusions on this issue. The majority of patients in the present series rapidly deteriorated neurologically or became septic, and therefore 22 patients underwent surgery within 24 hours after transferal to our department. The spinal cord is a very sensitive structure, so small compressive forces due to empyema can lead to devastating neurological deterioration. Therefore, we recommend early surgical treatment of cervical infections. Furthermore, surgical treatment should not only be used to harvest a bacteriological specimen, it should be used to achieve adequate debridement and restoration of stability.

In the past concerns have been raised about the ideal surgical technique. In degenerative cervical disc disease, the implantation of PEEK cages and expandable cylindrical cages is frequently performed with good clinical outcome results. Furthermore, concerns have been raised in the use of manufactured implants in cases of infection because of decrease antibiotic effectiveness, increased bac- 
terial adherence, glycocalyx formation, and the immune response. ${ }^{3,12,23}$ In 2006 Muzii et al. reported their results in a series of 8 patients with CSD and epidural empyema. All patients were treated with single-level anterior discectomy. No reconstruction of the disc space with either bone grafting or cage implantation was performed. The authors reported spontaneous bony fusion without a preserved alignment of the cervical spinal in all but one case. ${ }^{21}$

This technique of Muzi et al. is an interesting and very cost-effective approach to the problem, but in our opinion fusion of the two vertebral bodies using this technique might be associated with narrowing of the neural foramina, which could cause radicular pain. In the past decade several reports have demonstrated that the use of manufactured implants made of titanium and PEEK are not associated with chronic infections. ${ }^{19,20,36}$ In the present study, none of the patients who showed signs of latent or progressive infection on the MRI scan at follow-up visits. This finding supports the conclusion of other reports that chronic infections are not related to the implant. ${ }^{19}$ Furthermore, the 6-week antibiotic treatment in this series was not any longer than that of other reports and therefore did not suppresses the occurrence of a chronic infection.

However, the number of studies reporting on cervical infections is limited, and the surgical techniques, as well as the patient population, may vary considerably. The factors involving the surgical technique are the additional use of a cervical plate, the use of iliac crest graft versus intersomatic cage or corpectomy cage, and the use of posterior fixation. ${ }^{6,11,24,30,31}$ The rate of complications following the surgical treatment of cervical infections may vary from $16.66 \%$ to $33 \% .11,24,30,31$ Some authors even recommend avoidance of cervical plates because of implant loosening, with consecutive complications, and because of bacterial colonization of the implant. Cervical plating was performed in 26 of 29 patients (89.6\%), and indeed in one of those patients a repeat procedure was performed to treat implant loosening. However, in 1 of 3 patients (33.3\%) who underwent single-level ACDF, dislocation of the PEEK cage was noted and a repeat procedure was performed. In the present study, implant-related complications occurred in 4 patients $(13.8 \%)$. Since this is a retrospective study, it is difficult to identify the exact cause of implant dislocation in each case. Since all the repeat procedures were performed within the first 2 weeks after initial surgery, we believe that the poor bone quality caused by the infection was the reason for early implant dislocation. A closer look at the data reveals that implant dislocation and subsidence occurred in 2 of $3 \mathrm{ACDF}$ procedures without initial posterior fixation. Bicortical screw placement was not performed in any of the procedures in this series. Some might argue that bicortical screw placement would have increased the stability of the construct and could have prevented implant failure and eventually reoperation in some cases. However, we are of the opinion that monocortical screw placement is sufficient for ACDF. The poor bony quality and osteomyelitis are the most important factor for subsidence of PEEK cages and particularly of expandable cylindrical cages. Consecutive plate dislocation and screw loosening might be seen in some cases and might eventually be treated once the dislocation and loosening become symptomatic. We therefore recommend performing MRI and CT scanning to better plan out the procedure since these studies might help to identify patients with an increased risk of implant failure due to poor bony quality. We further recommend keeping the procedure (i.e., debridement) as short and small as reasonably practical. Partial corpectomy is a reasonable procedure in degenerative and trauma cases. However, due to the poor bony quality in cases of CSD and osteomyelitis, these principles do apply. Because early postoperative implant subsidence and dislocation have been frequently observed, posterior fixation should be performed as soon as possible once there is serious doubt about the fit of the implants, especially in ACCF procedures. This finding led to our adding posterior fixation to every ACCF procedure we performed for CSD.

\section{Conclusions}

The results of this study demonstrate that CSD is a serious disease. The first 2 weeks of treatment are critical, with a mortality rate of $10 \%$ and a repeat procedure rate of $20 \%$. An ICU stay of more than 3 days was required in $65 \%$ of the patients treated.

In addition to these major risks and complications in the perioperative period, the anterior approach is effective in the treatment of CSD. No recurrent or chronic infections were noted, $63.6 \%$ of patients were free of neck pain, and almost all patients with sensory and motor deficits showed resolution during follow-up. A larger prospective study is warranted to compare different surgical techniques and address the issue of surgical timing and the duration of antibiotic treatment.

\section{References}

1. Akbar M, Lehner B, Doustdar S, Fürstenberg CH, Hemmer $\mathrm{S}$, Bruckner T, et al: [Pyogenic spondylodiscitis of the thoracic and lumbar spine: a new classification and guide for surgical decision-making.] Orthopade 40:614-623, 2011 (Ger)

2. Al-Nammari SS, Lucas JD, Lam KS: Hematogenous methicillin-resistant Staphylococcus aureus spondylodiscitis. Spine (Phila Pa 1976) 32:2480-2486, 2007

3. Barth E, Myrvik QM, Wagner W, Gristina AG: In vitro and in vivo comparative colonization of Staphylococcus aureus and on orthopaedic implant materials. Biomaterials 10:325328, 1989

4. Berbari EF, Kanj SS, Kowalski TJ, Darouiche RO, Widmer AF, Schmitt SK, et al: 2015 Infectious Diseases Society of America (IDSA) Clinical Practice Guidelines for the Diagnosis and Treatment of Native Vertebral Osteomyelitis in Adults. Clin Infect Dis 61:e26-e46, 2015

5. Beronius M, Bergman B, Andersson R: Vertebral osteomyelitis in Göteborg, Sweden: a retrospective study of patients during 1990-95. Scand J Infect Dis 33:527-532, 2001

6. Bydon M, De la Garza-Ramos R, Macki M, Naumann M, Sciubba DM, Wolinsky JP, et al: Spinal instrumentation in patients with primary spinal infections does not lead to greater recurrent infection rates: an analysis of 118 cases. World Neurosurg 82:e807-e814, 2014

7. Curry WT Jr, Hoh BL, Amin-Hanjani S, Eskandar EN: Spinal epidural abscess: clinical presentation, management, and outcome. Surg Neurol 63:364-371, 2005

8. Darouiche RO: Spinal epidural abscess. N Engl J Med 355:2012-2020, 2006

9. Davis DP, Wold RM, Patel RJ, Tran AJ, Tokhi RN, Chan TC, 
et al: The clinical presentation and impact of diagnostic delays on emergency department patients with spinal epidural abscess. J Emerg Med 26:285-291, 2004

10. Digby JM, Kersley JB: Pyogenic non-tuberculous spinal infection: an analysis of thirty cases. J Bone Joint Surg Br 61:47-55, 1979

11. Ghobrial GM, Franco D, Theofanis T, Margiotta PJ, Andrews E, Wilson JR, et al: Cervical spondylodiscitis: presentation, timing, and surgical management in 59 patients. World Neurosurg 103:664-670, 2017

12. Gristina AG, Hobgood CD, Webb LX, Myrvik QN: Adhesive colonization of biomaterials and antibiotic resistance. Biomaterials 8:423-426, 1987

13. Heyde CE, Boehm H, El Saghir H, Tschöke SK, Kayser R: Surgical treatment of spondylodiscitis in the cervical spine: a minimum 2-year follow-up. Eur Spine J 15:1380-1387, 2006

14. Hlavin ML, Kaminski HJ, Ross JS, Ganz E: Spinal epidural abscess: a ten-year perspective. Neurosurgery 27:177-184, 1990

15. Karadimas EJ, Bunger C, Lindblad BE, Hansen ES, Høy K, Helmig P, et al: Spondylodiscitis. A retrospective study of 163 patients. Acta Orthop 79:650-659, 2008

16. Karikari IO, Powers CJ, Reynolds RM, Mehta AI, Isaacs RE: Management of a spontaneous spinal epidural abscess: a single-center 10-year experience. Neurosurgery 65:919-924, 2009

17. Korovessis P, Repantis T, Iliopoulos P, Hadjipavlou A: Beneficial influence of titanium mesh cage on infection healing and spinal reconstruction in hematogenous septic spondylitis: a retrospective analysis of surgical outcome of twenty-five consecutive cases and review of literature. Spine (Phila Pa 1976) 33:E759-E767, 2008

18. Krogsgaard MR, Wagn P, Bengtsson J: Epidemiology of acute vertebral osteomyelitis in Denmark: 137 cases in Denmark 1978-1982, compared to cases reported to the National Patient Register 1991-1993. Acta Orthop Scand 69:513-517, 1998

19. Kuklo TR, Potter BK, Bell RS, Moquin RR, Rosner MK: Single-stage treatment of pyogenic spinal infection with titanium mesh cages. J Spinal Disord Tech 19:376-382, 2006

20. Mondorf Y, Gaab MR, Oertel JM: PEEK cage cervical ventral fusion in spondylodiscitis. Acta Neurochir (Wien) 151:1537-1541, 2009

21. Muzii VF, Mariottini A, Zalaffi A, Carangelo BR, Palma L: Cervical spine epidural abscess: experience with microsurgical treatment in eight cases. J Neurosurg Spine 5:392-397, 2006

22. Nossaman VE, Larsen BE, DiGiacomo JC, Manuelyan Z, Afram R, Shukry S, et al: Mortality is predicted by Comorbidity Polypharmacy score but not Charlson Comorbidity Index in geriatric trauma patients. Am J Surg 216:42-45, 2018

23. Oga M, Sugioka Y, Hobgood CD, Gristina AG, Myrvik QN: Surgical biomaterials and differential colonization by Staphylococcus epidermidis. Biomaterials 9:285-289, 1988

24. Ozkan N, Wrede K, Ardeshiri A, Hagel V, Dammann P, Ringelstein A, et al: Cervical spondylodiscitis-a clinical analysis of surgically treated patients and review of the literature. Clin Neurol Neurosurg 117:86-92, 2014

25. Pereira CE, Lynch JC: Spinal epidural abscess: an analysis of 24 cases. Surg Neurol 63 (Suppl 1):S26-S29, 2005

26. Reihsaus E, Waldbaur H, Seeling W: Spinal epidural abscess: a meta-analysis of 915 patients. Neurosurg Rev 23:175-205, 2000
27. Ruf M, Stoltze D, Merk HR, Ames M, Harms J: Treatment of vertebral osteomyelitis by radical debridement and stabilization using titanium mesh cages. Spine (Phila Pa 1976) 32:E275-E280, 2007

28. Schimmer RC, Jeanneret C, Nunley PD, Jeanneret B: Osteomyelitis of the cervical spine: a potentially dramatic disease. J Spinal Disord Tech 15:110-117, 2002

29. Shad A, Shariff S, Fairbank J, Byren I, Teddy PJ, CadouxHudson TA: Internal fixation for osteomyelitis of cervical spine: the issue of persistence of culture positive infection around the implants. Acta Neurochir (Wien) 145:957-960, 2003

30. Shousha M, Boehm H: Surgical treatment of cervical spondylodiscitis: a review of 30 consecutive patients. Spine (Phila Pa 1976) 37:E30-E36, 2012

31. Shousha M, Heyde C, Boehm H: Cervical spondylodiscitis: change in clinical picture and operative management during the last two decades. A series of 50 patients and review of literature. Eur Spine J 24:571-576, 2015

32. Sørensen P: Spinal epidural abscesses: conservative treatment for selected subgroups of patients. Br J Neurosurg 17:513-518, 2003

33. Stüer C, Stoffel M, Hecker J, Ringel F, Meyer B: A staged treatment algorithm for spinal infections. J Neurol Surg A Cent Eur Neurosurg 74:87-95, 2013

34. Swanson AN, Pappou IP, Cammisa FP, Girardi FP: Chronic infections of the spine: surgical indications and treatments. Clin Orthop Relat Res 444:100-106, 2006

35. Urrutia J, Zamora T, Campos M: Cervical pyogenic spinal infections: are they more severe diseases than infections in other vertebral locations? Eur Spine J 22:2815-2820, 2013

36. Walter J, Kuhn SA, Reichart R, Kalff R, Ewald C: PEEK cages as a potential alternative in the treatment of cervical spondylodiscitis: a preliminary report on a patient series. Eur Spine J 19:1004-1009, 2010

37. Wang Z, Lenehan B, Itshayek E, Boyd M, Dvorak M, Fisher $\mathrm{C}$, et al: Primary pyogenic infection of the spine in intravenous drug users: a prospective observational study. Spine (Phila Pa 1976) 37:685-692, 2012

\section{Disclosures}

The authors report no conflict of interest concerning the materials or methods used in this study or the findings specified in this paper.

\section{Author Contributions}

Conception and design: Burkhardt, Oertel. Acquisition of data: Burkhardt, Müller, Wagner. Analysis and interpretation of data: all authors. Drafting the article: Burkhardt, Oertel. Critically revising the article: all authors. Reviewed submitted version of manuscript: Burkhardt, Oertel. Approved the final version of the manuscript on behalf of all authors: Burkhardt. Statistical analysis: Burkhardt, Oertel. Administrative/technical/material support: all authors. Study supervision: Burkhardt.

\section{Correspondence}

Benedikt W. Burkhardt: Klinik für Neurochirurgie, Universitätsklinikum des Saarlandes und Medizinische Fakultät der Universität des Saarlandes, Homburg-Saar, Germany. benedikt. burkhardt@gmail.com. 